ACCEPted By ApJ

Preprint typeset using $\mathrm{L}^{\mathrm{A}} \mathrm{T}_{\mathrm{E}} \mathrm{X}$ style emulateapj v. 11/10/09

\title{
RADIUS-EXPANSION BURST SPECTRA FROM 4U 1728-34: AN ULTRACOMPACT BINARY?
}

\author{
Duncan K. Galloway ${ }^{1}$, Yangsen YaO $^{2}$, Herman Marshall ${ }^{3}$, Zdenka Misanovic $^{1}$, Nevin Weinberg $^{4}$ \\ Accepted by ApJ
}

\begin{abstract}
Recent theoretical and observational studies have shown that ashes from thermonuclear burning may be ejected during radius-expansion bursts, giving rise to photoionisation edges in the X-ray spectra. We report a search for such features in Chandra spectra observed from the low-mass X-ray binary $4 \mathrm{U} 1728-34$. We analysed the spectra from four radius-expansion bursts detected in 2006 July, and two in 2002 March, but found no evidence for discrete features. We estimate upper limits for the equivalent widths of edges of a few hundred $\mathrm{eV}$, which for the moderate temperatures observed during the bursts, are comparable with the predictions. During the 2006 July observation 4U 1728-34 exhibited weak, unusually frequent bursts (separated by $<2 \mathrm{hr}$ in some cases), with profiles and $\alpha$-values characteristic of hydrogen-poor fuel. Recurrence times as short as those measured are insufficient to exhaust the accreted hydrogen at solar composition, suggesting that the source accretes hydrogen deficient fuel, for example from an evolved donor. The detection for the first time of a 10.77 min periodic signal in the persistent intensity, perhaps arising from orbital modulation, supports this explanation, and suggests that this system is an ultracompact binary similar to $4 \mathrm{U} 1820-30$.
\end{abstract}

Subject headings: stars: neutron - X-rays: binaries - X-rays: bursts - X-rays: individual $(4 \mathrm{U}$ 1728-34)

\section{INTRODUCTION}

One of the highest priorities for observational studies of neutron stars is measurement of the mass and radius, sufficient to constrain the uncertain equation of state (EOS; e.g. Lattimer \& Prakash 2007). A promising avenue to achieve such measurements is to detect surface spectral features from accreting neutron stars, which may preferentially show such features during thermonuclear (type-I) bursts, caused by unstable ignition of accreted $\mathrm{H} / \mathrm{He}$ on the surface of the neutron star. Identifying such features allows measurement of the surface redshift, and hence the compactness $(M / R$ ratio), which in turn allows constraints to be placed on the EOS. In recent years, only one claim for such features has been made (Cottam et al. 2002), and subsequent efforts have not been able to confirm this result (e.g. Cottam et al. 2008) [5. The majority of observational efforts to date have focussed on sources which show frequent bursts, which tend to be faint. Another class of bursts are significantly brighter, since the flux during the burst rise exceeds the Eddington limit (e.g Lewin et al. 1993), at which point the radiation pressure of the burst luminosity exceeds the local gravity. Recent theoretical work suggests that such bursts could drive a wind containing heavy-element ashes from the burning, which would imprint absorption features on the X-ray spectrum at the peak of the burst (Weinberg et al. 2006). Observa-

${ }^{4}$ University of California, Berkeley, CA 94720

${ }^{5}$ Additionally, the case for slow rotation in this system (required to produce lines as narrow as those observed) has weakened, with the detection of burst oscillations at $552 \mathrm{~Hz}$ (Galloway et al. 2010).
}

tional support for this hypothesis has been found from the low-resolution spectra of extremely intense "superexpansion" bursts. These bursts are the most intense of those thought to be powered by $\mathrm{H} / \mathrm{He}$, in contrast to the even longer, infrequent "superbursts", which are thought to be powered instead by carbon (e.g. Cumming et al. 2006). In superexpansion bursts observed by the Rossi $X$-ray Timing Explorer (RXTE), in 't Zand \& Weinberg (2010) found highly significant residuals for fits with a blackbody model. The residuals could be explained by absorption edge features, with energies consistent with highly-ionised iron-peak elements and depths indicative of abundances $\gtrsim 100$ times solar. However, the relatively poor spectral resolution prevents identification of the features.

Here we describe an attempt to detect and measure the $\mathrm{X}$-ray spectrum at the peak of radius-expansion bursts using the Chandra High energy Transmission Grating Spectrometer (HETGS). Few sources reliably show frequent, radius expansion bursts, and the key challenges for this search are the identification of a target and the observation triggering. One candidate is the globular cluster source $4 \mathrm{U} 1820-30$ which orbits in an ultracompact binary $\left(P_{\text {orb }}=685 \mathrm{~s}\right.$ Stella et al. 1987 $)$ and thus likely accretes almost pure helium. This system only exhibits bursts when in the minimum of its $\approx 180$-d intensity cycle (e.g. Priedhorsky \& Terrell 1984), and on one occasion was observed to produce 7 radius-expansion bursts separated by $193 \mathrm{~min}$ on average (Haberl et al. 1987). However, frequent bursts are not consistently observed, and recent pointed observations targeting the long-term minima have had limited success, for example detecting only four bursts in $1 \mathrm{Ms}$ of Rossi X-ray Timing Explorer observations (e.g. Gallowav et al. 2008). A better target is $4 \mathrm{U} 1728-34$, a well-known burst source for which the accretion rate does not reach such extreme values, and is typically $\sim 0.1 \dot{M}_{\text {Edd }}$. This rate is substantially lower 
(by almost an order of magnitude) than the maximum reached by $4 \mathrm{U} 1820-30$ and $4 \mathrm{U} 1728-34$ commonly produces radius-expansion bursts every $\approx 4-5 \mathrm{hr}$. The burst properties are otherwise similar to those of $4 \mathrm{U} 1820-30$ (Cumming 2003), and as we show here, it is possible that $4 \mathrm{U} 1728-34$ is also an ultracompact system.

\section{OBSERVATIONS \& ANALYSIS}

We observed 4U 1728-34 between 2006 July 17-23 with the HETGS (Canizares et al. 2005) aboard Chan$d r a$. The HETGS consists of two separate grating arrays (the Medium- and High-Energy Gratings, MEG and HEG) which intercept about $40 \%$ of the incoming photons and disperse them linearly along the Advanced CCD Imaging Spectrometer (ACIS). These instruments together provide spectra in the range 1.2-31 $\AA$ (10-0.4 $\mathrm{keV})$ with spectral resolution $(E / \Delta E)$ of up to 1000 , and wavelength resolution of up to $0.012 \AA$. We adopted the continuous clocking (CC) observing mode, for which the ACIS-S CCDs are read out continuously. This sacrifices one axis of spatial imaging but allows much higher incident photon countrates before photon pileup (multiple photons arriving at the same pixel in the same frame time) becomes a problem. Such mitigation strategies are necessary to deal with the highly variable incident count rates for a bursting source, but complicates the data reduction and spectral analysis. Specifically, CC-mode observations prevent the usual order sorting for dispersed spectra, which rely (in part) on both spatial axes, although the photon energy measured by the CCD allows unambiguous order sorting, at least for low dispersion orders. CC-mode observations also prevent extraction of the usual background spectrum.

We reduced the Chandra data with CIAO version 4.0 (December 2007; Fruscione et al. 2006), using the calibration database (CALDB) version 3.4.2. We undertook spectral fitting with ISIS version 1.4.9-4 (Houck \& Denicola 2000). We calculated response matrices (using MKGRMF) and ancilliary response files (using FULLGARF) for each observation. We also used the response and ancilliary files calculated for the entire observation to analyse the spectra from any bursts detected in those observations.

We also analysed archival Chandra timed exposure (TE) mode observations of 4U 1728-34 made on 2002 March 4, as well as archival XMM-Newton observations from 2002 October 29. We analysed the XMM-Newton data using the Science Analysis Software (SAS) version 7.0.0 (June 2006). We used data from the European Photon Imaging Camera (EPIC) pn CCD camera (Kuster et al. 2002), which was operated in timing mode. The EPIC pn camera is sensitive to photons in the range $0.15-15 \mathrm{keV}$, and with an effective area peaking at around $1400 \mathrm{~cm}^{2}$ at $2 \mathrm{keV}$. As with CC-mode for Chandra, in pn timing mode the central CCD alone is read out continuously, providing an effective time resolution of $0.03 \mathrm{~ms}$. The observations used in this paper are summarised in Table 1

\section{RESULTS}

We obtained in 2006 July three separate pointings of approximately 50, 150 and $40 \mathrm{ks}$ each, spanning almost 6 days, and detecting four, eighteen, and three bursts in each segment (respectively). We first analysed the persistent source spectrum between the bursts, to establish the persistent X-ray intensity. The raw lightcurve indicated significant variations in the persistent count rate from observation to observation, and also between each inter-burst interval. Thus, we divided each observation into segments delineated by each pair of bursts, excluding the intervals up to $150 \mathrm{~s}$ after each burst and $50 \mathrm{~s}$ before the next burst. For the first bursts of each pointing, we integrated from the beginning of the pointing to $50 \mathrm{~s}$ before the burst. We then fit the summed, firstorder MEG and HEG spectra simultaneously in the energy range $2.1-8.3 \AA(6-1.5 \mathrm{keV})$ with an absorbed comptonisation model, motivated by earlier broad-band spectroscopic measurements (see Misanovic et al. 2010, hereafter M10, for full details of the persistent spectral fitting, and references theirein for additional analyses of the persistent spectrum seen by Chandra). The neutral column density was fixed at $2.29 \times 10^{22} \mathrm{~cm}^{-2}$.

Like many low-mass X-ray binaries (LMXBs), $4 \mathrm{U}$ 1728-34 shows substantial variation in its X-ray spectrum and intensity on a range of timescales, from days to decades (e.g. Lewin et al. 1993), and the spectral "states" influence the burst properties. The integrated persistent flux during the 2006 Chandra observation was in the range $1.16-2.30 \times 10^{-9} \operatorname{ergs~cm}^{-2} \mathrm{~s}^{-1}(1.5-6 \mathrm{keV})$, equivalent to at least $3-5 \times 10^{-9} \mathrm{ergs} \mathrm{cm}^{-2} \mathrm{~s}^{-1}$ in the $2.5^{-}$ $25 \mathrm{keV}$ band, based on the extrapolated spectral model. These values are atypically high for the source; extensive earlier observations with $R X T E$ found the source in this flux range only $10 \%$ of the time (Galloway et al. 2008). In the absence of spectral information above $10 \mathrm{keV}$, it is difficult to identify the source spectral state; however, the nearest RXTE observation, on 2006 July 246, found a characteristically "soft" spectrum (e.g. with electron temperature for a compTT spectral component of $4.6 \mathrm{keV}$ ), and X-ray colors indicating the source was in the lower "island" region of the X-ray color-color diagram (see e.g. Galloway et al. 2008). The source flux was highest during the longest (second) pointing, consistently above $2.0 \times 10^{-9} \mathrm{ergs} \mathrm{cm}^{-2} \mathrm{~s}^{-1}(1.5-6 \mathrm{keV})$. In contrast to previous observations (e.g. D'Aí et al. 2006), no iron lines were detected in the persistent spectrum. For a distance to the source of $5.2 \mathrm{kpc}$ (from the peak flux of radiusexpansion bursts observed by RXTE; Galloway et al. 2008), and a bolometric correction which assumes a characteristic high-state "soft" spectrum (with electron temperature of $3 \mathrm{keV}$, c.f. with Falanga et al. 2006), the corresponding range of accretion rate is $4-7 \% 7$ of the Eddington rate $\left(\approx 1.8 \times 10^{18} \mathrm{~g} \mathrm{~s}^{-1}\right.$, assuming pure $\mathrm{He}$ accretion).

\subsection{The X-ray bursts}

We detected 25 bursts during the 2006 July observations (Fig. 11). The four bursts in the first observation (\#6568) were separated by $3.7 \pm 0.3 \mathrm{hr}$ on average, while in the second and third observations (\#6567, 7371 ) the mean recurrence time was significantly shorter,

6 Observation ID 92023-03-72-00

7 Note that the fraction will be correspondingly higher for nonzero $\mathrm{H}$-fraction, for example $6-11 \%$ of the Eddington rate $\dot{M}_{\text {Edd,H }}=10^{18} \mathrm{~g} \mathrm{~s}^{-1}$, assuming $\mathrm{H}$-fraction in the accreted fuel of $X_{0}=0.7$. 
TABLE 1

X-RAY OBSERVATIONS OF $4 \mathrm{U} 1728-34$

\begin{tabular}{|c|c|c|c|c|c|c|c|}
\hline No. & Date & Instrument & obsid & $\begin{array}{c}\text { Data } \\
\text { mode }\end{array}$ & $\begin{array}{c}\text { Exposure } \\
(\mathrm{ks})\end{array}$ & $\begin{array}{l}\text { No. of } \\
\text { bursts }\end{array}$ & Ref. \\
\hline 1 & 2002 Mar 4 & Chandra & 2748 & $\mathrm{TE}$ & 29.4 & 2 & {$[1]$} \\
\hline 2 & 2002 Oct 29 & XMM-Newton & 0149810101 & Timing $(\mathrm{PN})$ & 28.1 & 0 & \\
\hline 3 & $2006 \mathrm{Jul} 17-18$ & Chandra & 6568 & $\mathrm{CC}$ & 49.5 & 4 & {$[2,3$} \\
\hline 4 & 2006 Jul $18-20$ & Chandra & 6567 & $\mathrm{CC}$ & 151.8 & 18 & {$[2,3]$} \\
\hline 5 & $2006 \mathrm{Jul} 22-23$ & Chandra & 7371 & $\mathrm{CC}$ & 39.7 & 3 & {$[2,3]$} \\
\hline
\end{tabular}

References. - 1. D'Aí et al. (2006); 2. this paper; 3. Misanovic et al. (2010)

at $2.4 \pm 0.5 \mathrm{hr}$. The bursts themselves were short, with rise times of $\approx 1 \mathrm{~s}$ and lasting only $\approx 15 \mathrm{~s}$ (Fig. 2). The four bursts in the first segment (observation \#6568) were the brightest, with the bursts in the second and third segments reaching count rates only approximately $2 / 3$ the peak as in the first segment. A more detailed discussion of the burst properties and the inferred ignition conditions can be found in M10.

Two of the bursts in the first observation (\#6568) had indications of a constant intensity at maximum, lasting $\approx 1 \mathrm{~s}$. Since constant luminosity in the peak is an indication of radius-expansion, we carried out time-resolved blackbody spectral fits of the combined first-order HEG and MEG data for each set of bursts in each observation, to test for the characteristic variations in the blackbody radius and temperature expected for radius-expansion bursts. Following the conventional approach, we subtracted the pre-burst emission as background, and estimated the bolometric flux based on the blackbody spectral parameters. The average peak flux for the bursts in the three observations (\#6568, 6567 and 7371) was $8 \pm 2$, $7 \pm 2$ and $(5.9 \pm 1.9) \times 10^{-8} \operatorname{ergs~cm}^{-2} \mathrm{~s}^{-1}$, respectively. We found evidence for an elevated blackbody radius, coincident with a decrease in the blackbody temperature, only for the first four bursts (Fig. 3). While we did not fully resolve the rise in blackbody radius, this may largely have been due to the relatively low count rate early in the burst coupled with the steep rise time. These variations suggest that the first four bursts exhibited radiusexpansion. Although the confidence interval for the mean fluxes for the subsequent bursts were consistent with the maximum reached by radius-expansion bursts observed by other instruments (Galloway et al. 2003), the timeresolved spectral variations in those bursts gave no indications of radius-expansion.

We measured the ratio $\alpha$ (e.g. Gottwald et al. 1986) of the integrated persistent flux within each interval between the bursts, to the integrated flux of the subsequent burst (from time-resolved spectral fits; see M10) and obtained values which were 190 in the mean. The rapid timescales of the bursts, as well as the high $\alpha$-values, are both consistent with ignition of primarily He fuel. Although both the persistent flux and the burst recurrence times varied significantly, we found no correlation between the two, as might be expected (under the usual assumption that the persistent flux tracks the accretion rate). As discussed by M10, this lack of correlation may result from incomplete burning, or alternatively a varying area over which accretion takes place on the neutron star.

\subsection{Search for spectral features during the bursts}

We made a search for discrete features in the spectra from the bursts observed by Chandra in 2006 July. We first focussed on the four bursts observed in the first observation, \#6568, since only these exhibited any evidence for photospheric radius-expansion. We extracted spectra from various time intervals of each burst, and combined the \pm 1 orders to obtain a single first-order spectrum for each grating arm (HEG and MEG). We searched spectra extracted from 0-2 s (relative to the burst start time), and $0-4 \mathrm{~s}$. The combined total counts in HEG/MEG first-order for each of these intervals was 926/912 and $1740 / 1591$. We grouped the bins by various factors to obtain bin widths of $0.01,0.03,0.05,0.11$ and $0.22 \AA$, in order to search for both broad and narrow features. Since the counts per bin for these spectra was $<10$ at short and long wavelengths (away from the peak sensitivity) we adopted Gehrels weighting to determine errors on each bin, which better approximates Poisson uncertainties for low counts. This is preferable over the alternative approach, i.e. to bin adaptively to ensure some minimum number of counts per bin, since absorption features that saturate the spectrum and reduce the counts to zero will be missed. We fit each pair (HEG and MEG) of firstorder spectra using an absorbed blackbody with column density $n_{H}$ fixed at $2.3 \times 10^{22} \mathrm{~cm}^{-2}$ (derived from fits covering the first 2 seconds of each burst; see also M10). We set a detection threshold of $3-\sigma$, taking into account the total number of bins (HEG+MEG) in each time interval and binning.

We found no significant deviations from the blackbody (continuum) for any choice of binning, in either time interval. For the spectra extracted from the first two seconds of the bursts, which likely covers the entire radius expansion interval, we derive $3 \sigma$ upper limits for emission/absorption lines of equivalent width in the range 100-180 eV. We show the spectrum extracted over this interval in Figure 4, top panel.

We also estimated the upper limits on detection of absorption edges in the spectra. We added edge components to the model with the edge energy fixed at $\approx 3.1 \AA(4 \mathrm{keV})$, roughly where the observed count rate per bin of both HEG and MEG reached a maximum, in order to measure the best achievable limit with the available data. We found a $3 \sigma$ upper limit of 0.26 (0.20) on the optical depth $\tau$ for the $0-2 \mathrm{~s}(0-4 \mathrm{~s})$ spectra respectively, corresponding to equivalent widths of $280(250) \mathrm{eV}$. We also searched spectra from after the radius expansion episode, in case the photosphere was too 

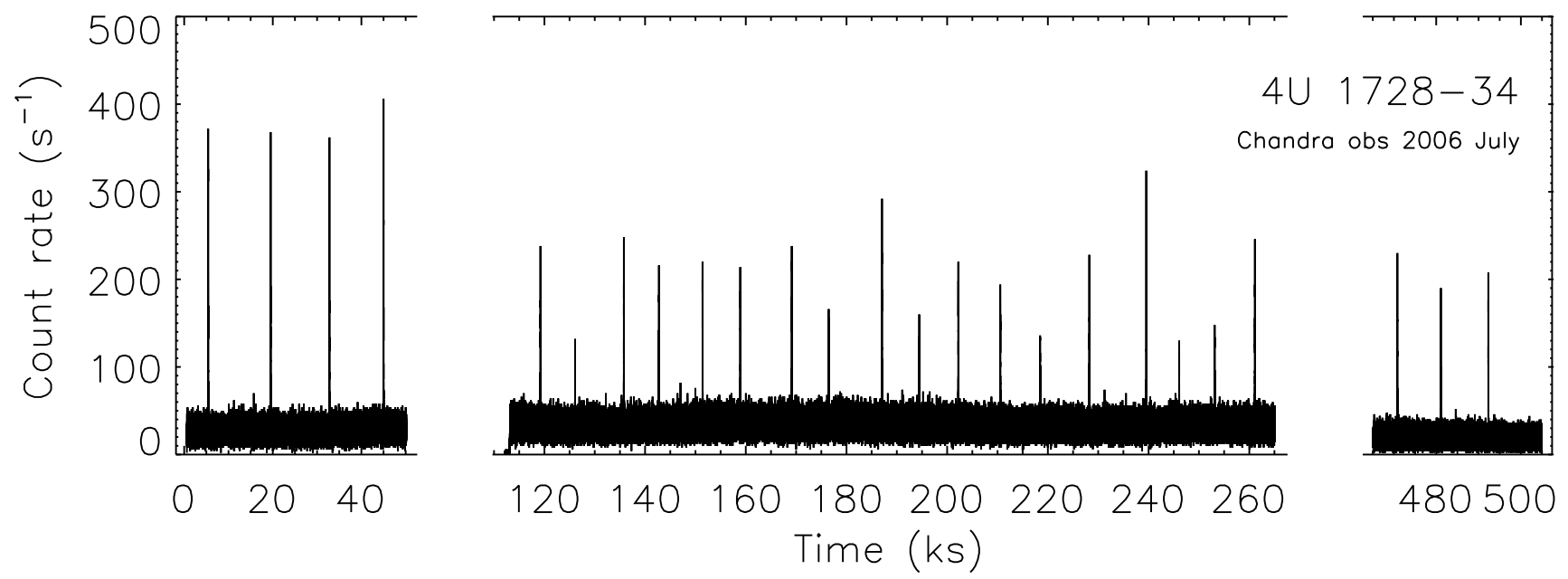

Fig. 1. - The 2006 July Chandra observation of $4 \mathrm{U} 1728-34$, showing the 25 thermonuclear bursts detected, which were separated by recurrence times in the range $1.82-3.92 \mathrm{hr}$. The first-order photons dispersed by the HETGS were combined and binned at $0.5 \mathrm{~s}$ time resolution; the lightcurve for the zeroth-order (undispersed) photons was dominated by pileup, which resulted in truncation of the peaks of the bursts, so these photons were excluded.

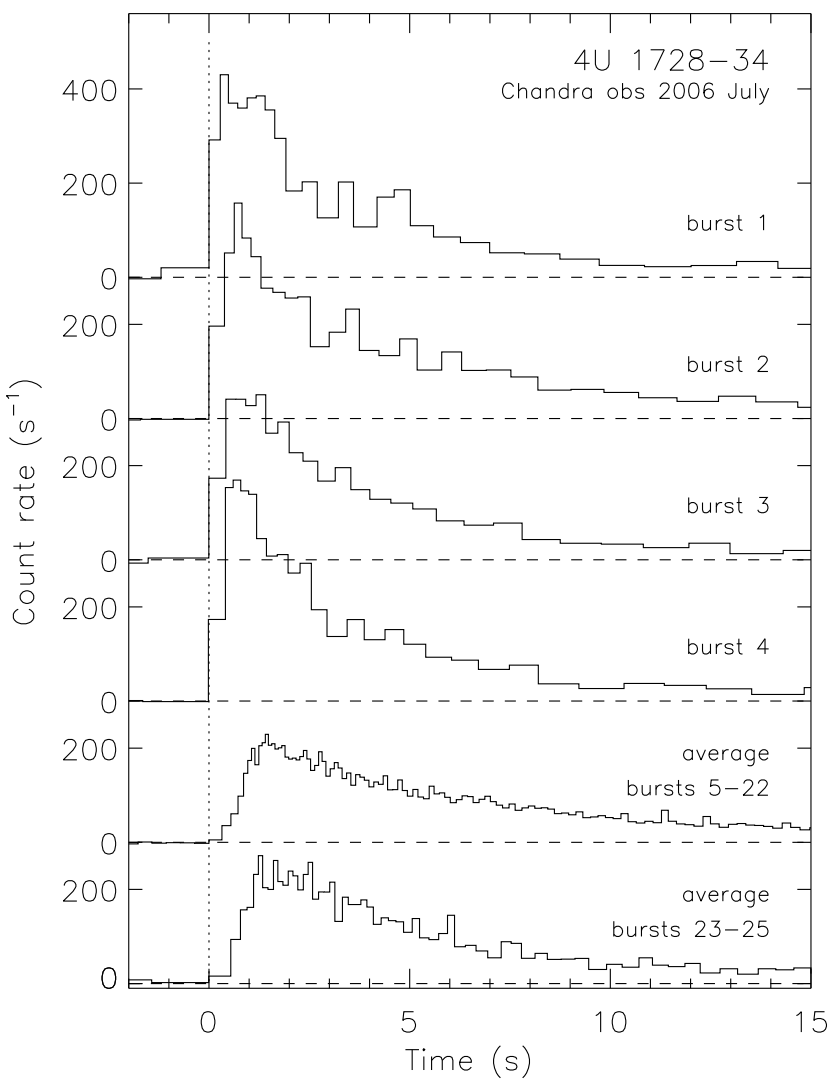

FIG. 2.- Burst profiles from the three intervals comprising the 2006 July Chandra observation of $4 \mathrm{U} 1728-34$. The first four bursts, from observation 6568 , are plotted individually; we subtract the pre-burst emission (dashed lines) and shift the profiles vertically for clarity. Dispersed events were binned to ensure 100 counts per bin. We also plot the combined lightcurves from second and third observations, 6567 and 7371 , binned with 400 and 100 counts per bin, respectively. Note the markedly different peak intensity and shape of bursts 1-4 compared to those that followed.

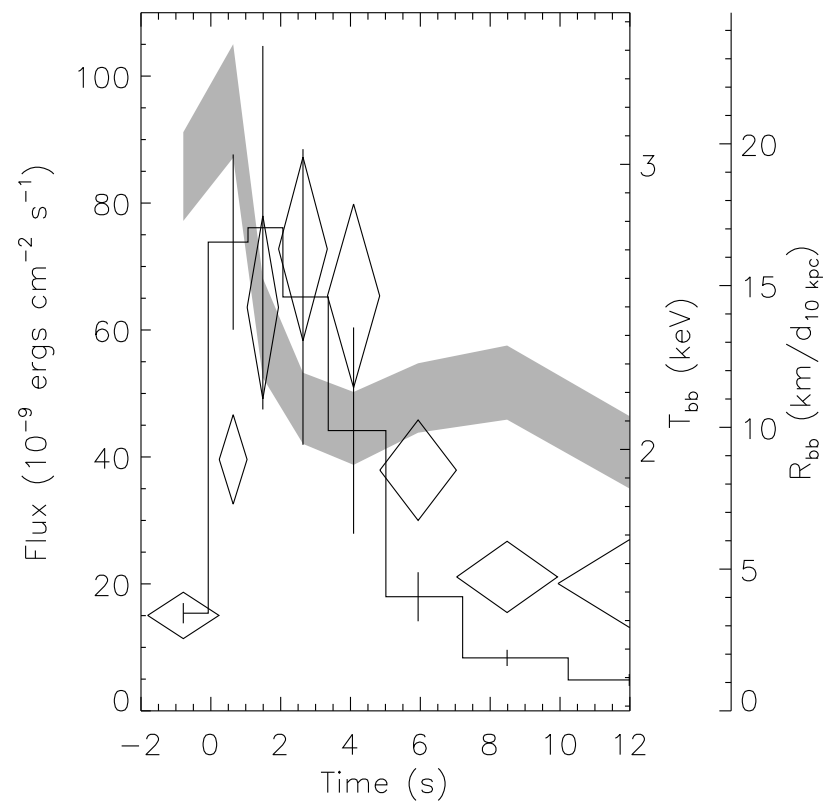

Fig. 3.- Time-resolved spectroscopy of the summed signal for the first four bursts, detected in observation \#6568. The histogram shows the (bolometric) burst flux (left-hand $y$-axis) in units of $10^{-9} \mathrm{erg} \mathrm{cm}^{-2} \mathrm{~s}^{-1}$, with error bars indicating the $1 \sigma$ uncertainties. The grey ribbon shows the $1 \sigma$ limits of the blackbody radius (outer right-hand $y$-axis) in $\mathrm{km} / d_{10 \mathrm{kpc}}$. The diamonds show the $1 \sigma$ error region for the blackbody temperature (inner right-hand $y$-axis) in keV. Note the elevated blackbody radius (to approximately twice the value in the tail) during the second time-bin near the burst peak. This radius increase indicates photospheric radiusexpansion in these bursts.

hot during radius-expansion for neutral or incompletelyionised species to survive. In spectra extracted from time window covering $2-16$ and $4-16 \mathrm{~s}$ after the burst start, we obtained upper limits as before on edges of 250 and $260 \mathrm{eV}$, respectively.

Although the bursts observed in the second and third observations (\#6567 and 7371) likely did not exhibit radius-expansion, we undertook a second search of spec- 

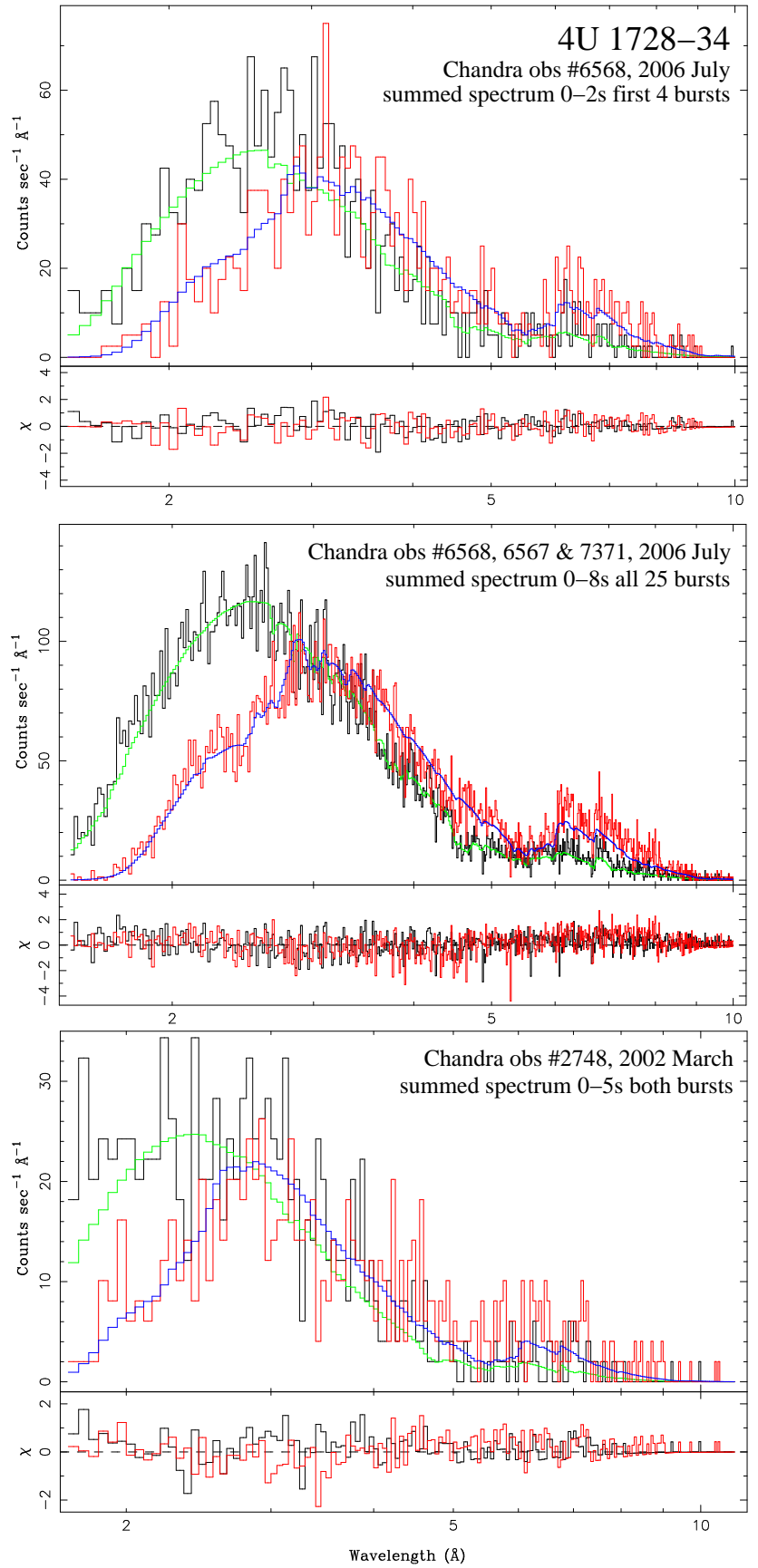

FIG. 4.- HEG and MEG first-order spectra extracted from thermonuclear bursts observed by Chandra. The top panel shows the spectra extracted from 0-2 s (relative to the burst start) from the first four bursts of observation \#6568, in 2006 July, and binned at $0.11 \AA$. The summed \pm 1 order HEG and MEG are shown separately (black and red histograms), as well as the best-fitting blackbody model (green and blue). The middle panel shows the spectra extracted from 0-8 s of all 25 bursts observed by Chandra in 2006 July, binned at $0.03 \AA$. Note the feature around $5.28 \AA$, which is closest to our $3 \sigma$ significance threshold, taking into account the number of spectral bins. The bottom panel shows the spectra extracted from $0-5$ s of the two bursts observed by Chandra in 2002 March (observation \#2748). tra extracted from a wider choice of time intervals (relative to the start) of all 25 bursts. The combined total counts in HEG/MEG first-order for each of these intervals, i.e. $0-2,0-4,0-8$ and $0-16 \mathrm{~s}$, as well as $2-16,4-$ 16 and $8-16$ s was $3540 / 3350,7670 / 7180,12880 / 12470$, $18680 / 19190$, respectively. We found no broad or narrow features that exceeded our $3 \sigma$ confidence threshold for any choice of time interval or binning. The most significant deviation was in the MEG spectra extracted from $0-8$ s of all 25 bursts, and binned at $0.03 \AA$, in which a single bin around $5.28 \AA$ was below the model fit at a significance of almost $3 \sigma$ (Fig. 4, middle panel). No similar feture was seen in the HEG spectrum.

Despite several attempts in recent years, observations of radius-expansion bursts by Chandra or XMM-Newton have eluded observers. In fact, we are aware of only one other confirmed radius-expansion burst, which was detected on 2002 March 4 22:42:45 (MJD 52337.94637) in observation \#2748 (Table 11). This burst was also detected by $R X T E$ (burst \#101 in Galloway et al. 2008), and was found to exhibit photospheric radius expansion. This event was preceded by a similar burst at UT 18:35:24 (MJD 52337.77459), $4.12 \mathrm{hr}$ earlier. Under the assumption that this burst too reached the Eddington limit, we extracted spectra from the peak and the tail of each burst, and combined them to search for discrete spectral features.

Unlike the observations in 2006 July, observation \#2748 was performed in Timed Exposure (TE) mode, with the CCDs read out every $1.44 \mathrm{~s}$. The extreme brightness of the source during the radius expansion bursts likely led to substantial pileup during the bursts, although this was not obvious from the dispersed burst spectra. We combined spectra from the HEG and MEG \pm 1 order from the first five and next 14-19 seconds of the two bursts, and examined the spectra for any evidence of discrete features. Due to the small number of total counts in the two sets of spectra (600-800 counts total in each grating arm), we rebinned the HEG (MEG) by a factor of 20 (10) to achieve a bin size of approximately $0.11 \AA$. We obtained an acceptable fit using an absorbed blackbody model, and found no discrete features which exceeded our $3 \sigma$ detection threshold (taking into account the number of spectral bins). A representative spectrum, from the first five seconds of the two bursts summed, is shown in Fig. 4 (bottom panel). We estimate $3 \sigma$ upper limits on the equivalent width of discrete features of $\pm 150 \mathrm{eV}$.

\subsection{Persistent intensity variations}

The long, uninterrupted X-ray lightcurve accumulated during the Chandra observation allowed a search for periodic signals with sensitivity greater than for any other dataset, particularly at low energies. Persistent X-ray intensity variations in LMXBs are sometimes observed at the orbital period, which for 4U 1728-34 remains unknown. We concentrated on the longest (second) segment of data, and created a lightcurve from first-order photons (both HEG and MEG) in the $\approx 6-25 \AA(2-$ $0.5 \mathrm{keV}$ ) energy range (where orbital variations of neutral absorption would be expected to have the largest effect) at $60 \mathrm{~s}$ time resolution. We removed the 1-2 min segments including each burst, and filled the resulting 


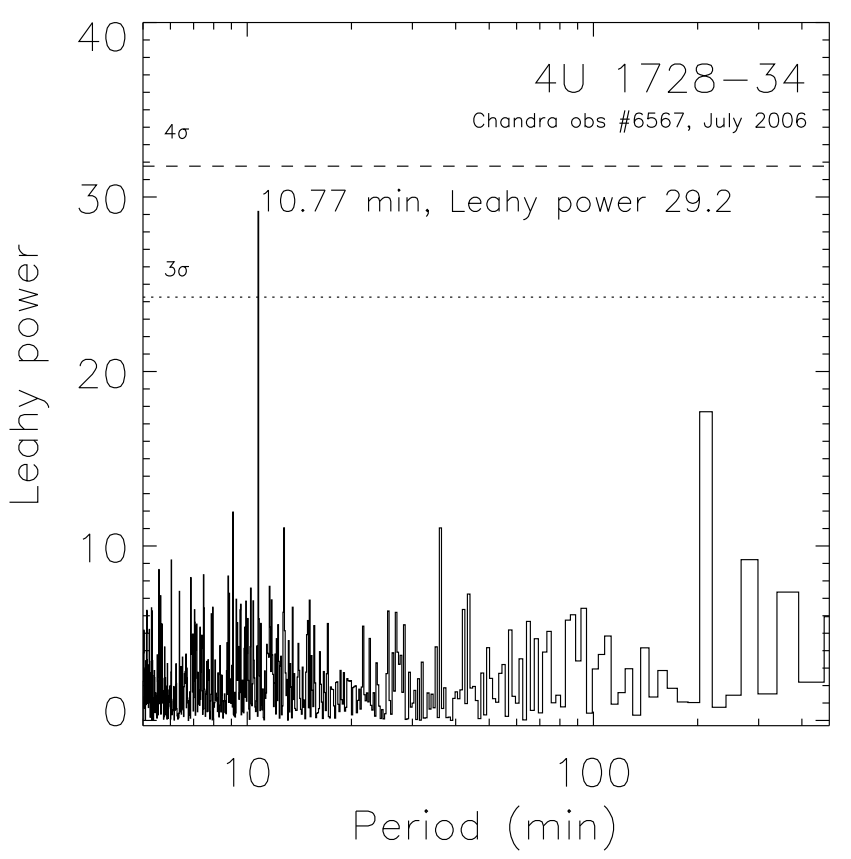

FIG. 5.- Power density spectrum for the longest contiguous segment of the 2006 Chandra observation of $4 \mathrm{U} 1728-34$. The 3 - and 4- $\sigma$ detection thresholds (taking into account the number of bins in the frequency range of interest) are indicated, as is the bin with the maximum power, at a period of $10.77 \mathrm{~min}$.

gaps with normally-distributed values consistent with the local mean and standard deviation. The overall mean countrate for the lightcurve was $(8.0 \pm 0.4)$ count $\mathrm{s}^{-1}$.

The Fourier power spectrum of the resulting light curve exhibited a peak with Leahy power of 25-29 at 10.77 min, significant (taking into account the number of trials) at between 3 and $4 \sigma$ (i.e. at greater than $99.7 \%$ significance; Fig. 5). The peak power and significance vary because of the use of random values to fill the gaps in the lightcurve where the bursts were removed, but the detection was consistently $>3 \sigma$ over many trials. The signal was approximately sinusoidal, with an RMS amplitude of $0.5 \%$. We searched for the 10.77 min signal in the full-range lightcurve, as well as other energy bands. The Leahy power at the corresponding frequency in the FFTs from the full-range lightcurve was in the range $17-20$, indicating $\mathrm{a} \approx 2 \sigma$ detection. There was evidence that the signal power decreased with energy above $2 \mathrm{keV}$; nominal power was detected both in the 2-3 and $3-4 \mathrm{keV}$ bands, with corresponding 3- $\sigma$ upper limits on the RMS amplitude of 0.4 and $0.3 \%$, respectively. A stronger signal with Leahy power $\approx 40$ was detected in the $3-4 \mathrm{keV}$ lightcurve at a period of $16.64 \mathrm{~min}$, very close to the spacecraft yaw dither period of $1000 \mathrm{~s}$, and so likely instrumental. We also searched a lightcurve comprising the zeroth-order photons in the $<2 \mathrm{keV}$ band. The count rate in the undispersed low-energy photons was just 2.2 count $\mathrm{s}^{-1}$ in the mean, substantially less than that of the first-order photons, likely due to pileup. We detected no significant power at the $10.77 \mathrm{~min}$ period, deriving an upper limit on the rms amplitude of $0.7 \%$, which cannot rule out the detection in the dispersed photon lightcurve.

Several other archival observations of 4U 1728-34 are available, and we also undertook timing analyses of some of these data in an attempt to confirm the 10.77 min pe- riod. The earlier Chandra observation on 2002 March 4 (observation ID \#2748, Table 1] see also D'Aí et al. 2006) found the source at a significantly lower intensity, and the first-order count rate below $2 \mathrm{keV}$ was around 5.3 count $\mathrm{s}^{-1}$. We computed a 60 -s lightcurve omitting the intervals surrounding the two bursts (see 3.2 ), and filled the resulting gaps with random values as with the 2006 data. No signal was detected at the candidate period. With the observation only lasting $9.1 \mathrm{hr}$, the corresponding upper limit on the candidate signal amplitude was $1.7 \%(3 \sigma)$. An XMM-Newton observation on 2002 October 29 (observation ID \#0149810101, Table 1) was similarly substantially shorter than the 2006 Chandra observation, at only $7.5 \mathrm{hr}$. We calculated a lightcurve by accumulating photons with energies $\leq 2 \mathrm{keV}$ in 60 -s bins, with a mean rate of 9.4 count $\mathrm{s}^{-1}$. We found no signal at the candidate period, and derive a $3 \sigma$ upper limit on the rms amplitude of $0.8 \%$. As with the earlier Chandra data, this is above the detected amplitude of the signal in the 2006 observations.

\subsection{The $X$-ray position and optical counterpart}

The detection of a short-period signal in the persistent X-ray emission during the 2006 observation led us to reexamine the optical counterpart proposed by Martí et al. (1998). The infrared magnitudes combined with the measured column density lead those authors to suggest a counterpart "less luminous and less massive than a middle B/early A V star or a F-K III star", taking into account the substantial contribution of the accretion disk. Here we investigate whether the observations are consistent with an ultracompact counterpart similar to that of the similar 10-min binary 4U 1820-30.

The counterpart to that system was detected in the $B$ and F140W UV bands by King et al. (1993). The ratio of the absorption-corrected fluxes in the two bands was approximately $(\Delta \lambda / \lambda)^{-3.2}$, so that the spectral slope was close to the Rayleigh-Jeans value. We extrapolated the observed spectrum to the $J$ and $K$ bands, and corrected for the different distances of $7.6 \mathrm{kpc}$ for $4 \mathrm{U} 1820-30$ and $5.7 \mathrm{kpc}$ for $4 \mathrm{U} 1728-34$ (assuming that radius-expansion bursts reach the Eddington limit for a helium atmosphere; Galloway et al. 2003). The expected magnitudes at the distance of $4 \mathrm{U} 1728-34$ are $J=17.5$ and $K=17.3$, which are substantially different to the absorption-corrected values of 19.6 and 15.1 measured by Martí et al. (1998). On the other hand, if we extrapolate using a spectral slope of 4 , we expect $J=19.4$ and $K=19.7$, which at least matches the $J$-band luminosity for the proposed counterpart. We conclude that a counterpart similar to $4 \mathrm{U}$ 1820-30 may give a consistent $J$-band luminosity to the proposed $4 \mathrm{U}$ 1728-34 counterpart, but the measured $K$-band brightness is too high. This may indicate an additional IR component as has been inferred to exist in other compact binaries (e.g. Greenhill et al. 2006).

Alternatively, we consider the possibility that the proposed counterpart is unrelated and positionally coincident with $4 \mathrm{U} 1728-34$ by chance. First we determined an accurate X-ray position from the Chandra observation \#2748 (Table 11). The zeroth-order image for this bright source was significantly piled up, leaving a ring of emission rather than the point source usual for fainter sources. The standard source detection algorithms can- 
not provide a precise position for such images, so instead we fit the position of the dispersed HEG and MEG spectra, as well as the readout trace (an artifact arising from the shuffling of charge along the CCD illuminated by a bright point source) and computed their intersection. We first checked for, and corrected, an aspect offset of -0.46 in R.A. and $-1^{\prime \prime} .03$ in Ded 8 . We determined a position for $4 \mathrm{U} 1728-34$ of $R . A .=17^{\mathrm{h}} 31^{\mathrm{m}} 57^{\mathrm{s}} .69$, decl. $=-33^{\circ} 50^{\prime} 01^{\mathrm{s}} .3(\mathrm{~J} 2000.0)$, with an estimated $90 \%$ confidence uncertainty of 0.6 (corresponding to the systematic pointing uncertainty for Chandra). We note that this position is within 1.! 3 of the radio source (Martí et al. 1998), confirming the positional coincidence of the radio and X-ray sources.

Finally, we estimated the field density of stars with $K \leq 15$ based on the IR star count model of Nakajima et al. (2000). We calculate a total of 280 objects per $\operatorname{arcmin}^{2}$ towards $4 \mathrm{U}$ 1728-34, which suggests a reasonable likelihood of chance positional alignment within an arcsec. Thus, we cannot place any significant constraints on the nature of $4 \mathrm{U} 1728-34$ based on the properties of the proposed counterpart.

\section{DISCUSSION \& CONCLUSIONS}

The 2002 and 2006 Chandra observations represent the only high-spectral resolution observations of radiusexpansion bursts from any source to date. Only six such bursts have been observed in total, and the data from two of those bursts (from observation \#2748) likely suffer from pileup as well as having too poor time resolution to resolve the radius-expansion episode. Nevertheless, this work represents the first such probe into a poorlystudied burst spectral regime. We detected no evidence for discrete features in the spectra extracted from during the radius-expansion episodes, and derive upper limits for narrow features of approximately $150 \mathrm{eV}$.

The super-Eddington flux that drives the radius expansion during these bursts transforms nearly all the excess luminosity into kinetic and potential energy of the extended atmosphere. The radiation-driven winds which result are capable of expelling $\gtrsim 0.5 \%$ of the accreted mass (e.g. Hanawa \& Sugimoto 1982; Nobili et al. 1994). If a fraction of the heavy elements produced during burning are expelled in a wind and exposed at the surface, we may expect to observe photoionisation edges from these elements.

However, we found no evidence for photoionization edges, and derive typical upper limits of $250-300 \mathrm{eV}$. The strength of edge features depends upon the elemental abundances in the burning products, the temperature and degree of expansion of the photosphere (that sets the degree of ionisation) and the amount of material ejected (that determines the column depth; e.g. in 't Zand \& Weinberg 2010). In order to estimate the likely equivalent widths, we adopted model predictions from Weinberg et al. (2006). We assumed abundances in the burning products for the pure-He model (see e.g. Fig. 13 of (Weinberg et al. 2006) ), since the burst profiles and other evidence presented here suggest pure He accretion. The abundance distributions may vary if there is

\begin{tabular}{|c|c|c|c|c|c|c|}
\hline $\begin{array}{c}8 \\
\text { used }\end{array}$ & $\begin{array}{l}\text { This } \\
\text { data }\end{array}$ & $\begin{array}{l}\text { correction } \\
\text { processed }\end{array}$ & $\begin{array}{l}\text { was } \\
\text { prior }\end{array}$ & $\begin{array}{c}\text { required } \\
\text { to }\end{array}$ & $\begin{array}{l}\text { since } \\
2004 .\end{array}$ & we \\
\hline
\end{tabular}

hydrogen present in the ignition layer. We note that the rp-process burning that would occur if $\mathrm{H}$ is present (e.g. Schatz et al. 2001) occurs after the radius expansion, so heavy burning products will not be ejected in the wind, unless they get dredged up from the ashes of previous bursts. in 't Zand \& Weinberg (2010) found evidence for absorption edges with optical depths of $\tau=0.2-3$ in superexpansion bursts, in which the photospheric radius increases to $>1000 \mathrm{~km}$. The expansion in the bursts from $4 \mathrm{U}$ 1728-34 was much more modest, at around $20 \mathrm{~km}$ (a factor of $\approx 2$ above the NS surface; Fig. 3 ), and it is possible that this distinction is the reason for the nondetection in our data. However, we note that the upper limits on optical depth that we derive, of $\tau \approx 0.2-0.3$, are consistent with the measured values for the weaker edges in the superexpansion bursts. Thus, for moderate expansion bursts such as those seen from $4 \mathrm{U}$ 1728-34, we can rule out the strongest edges detected in the superexpansion bursts, but not the weakest.

The predicted column density and equivalent widths for selected elements are shown in Fig. 6. For comparison, we calculated the effective temperature in the neutron-star photosphere, adopting a correction to the observed (color) temperature of 2 (see e.g. Madei et al. 2004) and a surface gravitational redshift of 1.31 (equivalent to a $1.41 M_{\odot}$ neutron star with $R=10 \mathrm{~km}$ ), that have the net result of reducing the observed (color) temperature by a factor of 1.5 to give the effective temperature in the neutron star frame. We calculated the effective temperatures both at the time of maximum expansion of the photosphere, and also at the point where the photosphere is assumed to have rejoined the neutron star surface ("touchdown"). The detectability of edge features is strongly dependent upon the effective temperature in the derived range, between 1 and $1.8 \mathrm{keV}$. Interestingly, the predicted equivalent width for $\mathrm{S}$ and $\mathrm{Si}$ at an effective temperature of $\approx 1 \mathrm{keV}$ are a few hundred to a thousand $\mathrm{eV}$, comparable to the upper limits on detection derived in the present spectra. Doppler broadening due to rotation may reduce the detectability of the edges in moderate radius expansion bursts. If angular momentum in the expanding photosphere is conserved, the transverse velocity of the expanded shell in the bursts from $4 \mathrm{U}$ 1728-34 would be reduced by a factor of two, such that the degree of Doppler broadening would be equivalent to a source rotating at $\approx \nu_{\text {spin }} / 2=181 \mathrm{~Hz}$. The reduction in detectability for an intrinsically broad feature such as an edge will not be so severe as for spectral lines (e.g. Chang et al. 2006), although it may be significant. We hope that future observations, that will contribute additional photons to the already-accumulated spectrum emitted at the peak of radius-expansion bursts, will allow us to make more sensitive searches for spectral features.

The properties of the bursts observed from 4U 1728-34 by Chandra suggest that the fuel contains little or no hydrogen. During the 2006 July observation the source entered a state of unusually high accretion rate, so that (from the second observation, \#6567 onwards) the burst ignition conditions were reached before sufficient fuel to power a radius-expansion burst had accumulated. The occurrence of bursts with characteristic He-rich profiles and high $\alpha$-values at such short recurrence times are difficult to understand based on standard burst theory. For 


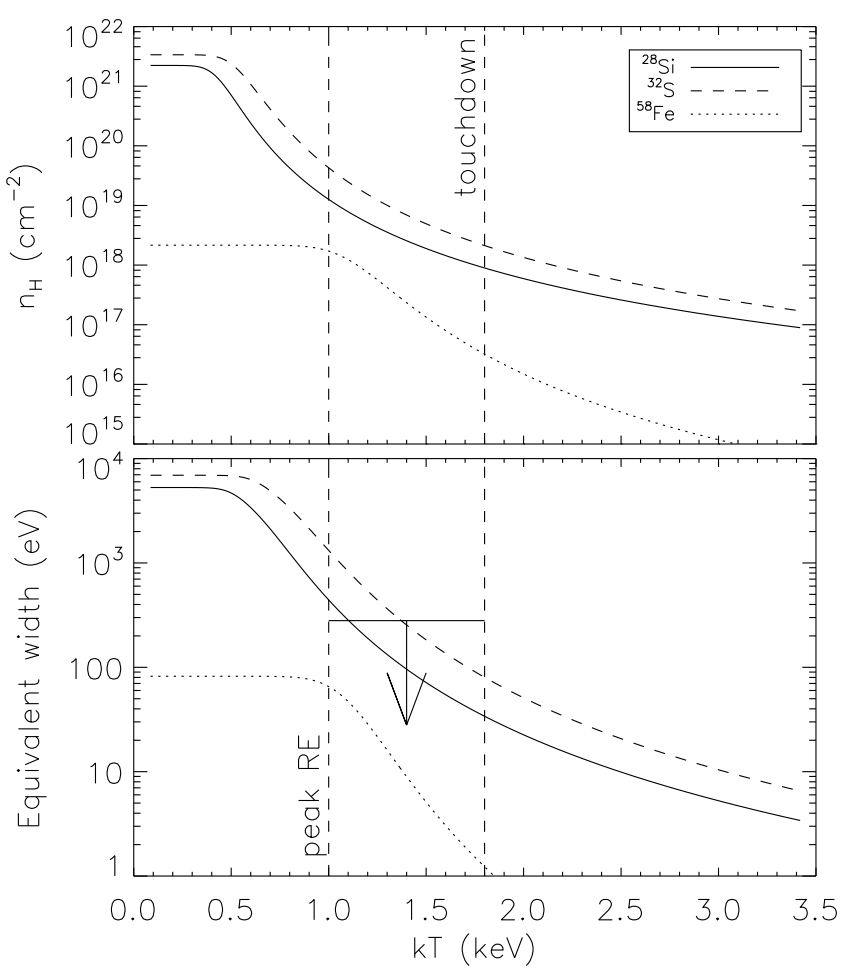

FIG. 6.- Column density (top panel) and edge equivalent width (bottom panel) for representative elements predicted for model He0.1 (pure He accretion at $0.1 \dot{M}_{\text {Edd }}$ ) of Weinberg et al. (2006), as a function of effective temperature. The estimated effective temperature at the time of maximum radius expansion and subsequent touchdown during the first four bursts observed by Chandra in June 2006 are indicated (vertical dashed lines). The upper limit on edge depth derived for the summed spectra from four radiusexpansion bursts from $4 \mathrm{U} 1728-34$ is indicated.

a neutron star accreting mixed $\mathrm{H} / \mathrm{He}$ at around $10 \%$ the Eddington rate (within the range in which we observed $4 \mathrm{U}$ 1728-34), $\mathrm{H}$ will burn stably between the bursts, reducing the fraction in the fuel at ignition. However, the burst recurrence time observed in 2006 July is insufficient to exhaust the $\mathrm{H}$ for solar abundances. Steady (hot CNO) H-burning is mediated by $\beta$-decays which limit the rate, so that the accreted hydrogen will only be exhausted at the ignition depth in a time $t=11\left(X_{0} / 0.7\right)\left(Z_{\mathrm{CNO}} / 0.02\right)^{-1} \mathrm{hr}$, where $X_{0}$ is the hydrogen fraction in the accreted material and $Z_{\mathrm{CNO}}$ is the mass fraction of $\mathrm{CNO}$ nuclei (0.7 and 0.02 respectively, for solar composition). For burst recurrence times of $\approx 2 \mathrm{hr}$ there is insufficient time between bursts to reduce the $\mathrm{H}$-fraction in the fuel significantly below the accreted level prior to burst ignition. If the source were accreting hydrogen at approximately solar composition, the expected burst profiles would be much longer, with $\approx 5 \mathrm{~s}$ rises and tails out to $100 \mathrm{~s}$, similar to what has been observed for GS 1826-24 (Galloway et al. 2004). In those bursts the longer timescale comes from the much slower progression of $\beta$-decay mediated rp-process $\mathrm{H}$ burning. We suggest three possible explanations for the burst properties. First, that the source accretes material which is unusually rich in $\mathrm{CNO}$ nuclei. With $Z_{\mathrm{CNO}}$ approximately 5 times in excess of the solar value, the time to exhaust all the $\mathrm{H}$ would be of order the burst recurrence time observed during the 2006 Chandra observation. There is no observational support for this suggestion, as the optical counterpart for the source has not been confirmed, so the type of the mass donor is not known. Second, it is possible that shear-mediated mixing allows CNO nuclei from the ashes of previous bursts to be dredged up into the freshly accreted material, as has been predicted for slowly rotating neutron stars by Piro \& Bildsten (2007). 4U 1728-34 is known to be rotating at $363 \mathrm{~Hz}$ (Strohmaver et al. 1996), which is fairly slow for accreting neutron-stars in LMXBs, but the full effects of such mixing in sources accreting hydrogen as well as helium have not yet been fully investigated. Either of these possibilities leave open the chance of long $\mathrm{H}$-rich bursts occurring occasionally from the source, although these have not yet been observed. Continued observations, as well as an archival search underway with data from multiple instruments, will test these hypotheses.

Third, it remains possible that the source accretes Hpoor material, from an evolved (e.g. white dwarf) donor. Such systems are referred to as ultracompact binaries, due to their typically very short $(<80 \mathrm{~min})$ orbital periods (e.g. in 't Zand et al. 2007). The 10.77 min modulation in the intensity of low-energy X-rays detected for the first time by Chandra from 4U 1728-34 supports this possibility. If this modulation was orbital, the system would be essentially a twin of $4 \mathrm{U} 1820-30$. Like that source, $4 \mathrm{U}$ 1728-34 will be detectable with the ESA/NASA Laser Interferometer Space Antenna (LISA; Nelemans 2008). Existing measurements of the candidate IR counterpart cannot significantly constrain this possibility, and deeper infrared observations are required to unambiguously confirm the ultracompact nature of this source, via detection of a corresponding modulation in the counterpart intensity.

This research has made use of data obtained from the Chandra Data Archive and the Chandra Source Cata$\log$, and software provided by the Chandra X-ray Center (CXC) in the application package CIAO. We thank D. Kaplan for supplying the code to estimate star counts in the 4U 1728-34 field.

Facilities: CXO(ASIS), XMM(EPIC)

\section{REFERENCES}

Canizares, C. R., Davis, J. E., Dewey, D., Flanagan, K. A., Galton, E. B., Huenemoerder, D. P., Ishibashi, K., Markert, T. H., Marshall, H. L., McGuirk, M., Schattenburg, M. L., Schulz, N. S., Smith, H. I., \& Wise, M. 2005, PASP, 117, 1144 Chang, P., Morsink, S., Bildsten, L., \& Wasserman, I. 2006, ApJ, 636, L117

Cottam, J., Paerels, F., \& Mendez, M. 2002, Nature, 420, 51
Cottam, J., Paerels, F., Méndez, M., Boirin, L., Lewin, W. H. G., Kuulkers, E., \& Miller, J. M. 2008, ApJ, 672, 504

Cumming, A. 2003, ApJ, 595, 1077

Cumming, A., Macbeth, J., Zand, J. J. M. i., \& Page, D. 2006, ApJ, 646, 429

D’Aí, A., di Salvo, T., Iaria, R., Méndez, M., Burderi, L., Lavagetto, G., Lewin, W. H. G., Robba, N. R., Stella, L., \& van der Klis, M. 2006, A\&A, 448, 817 
Falanga, M., Götz, D., Goldoni, P., Farinelli, R., Goldwurm, A., Mereghetti, S., Bazzano, A., \& Stella, L. 2006, A\&A, 458, 21

Fruscione, A., McDowell, J. C., Allen, G. E., Brickhouse, N. S., Burke, D. J., Davis, J. E., Durham, N., Elvis, M., Galle, E. C., Harris, D. E., Huenemoerder, D. P., Houck, J. C., Ishibashi, B., Karovska, M., Nicastro, F., Noble, M. S., Nowak, M. A.,

Primini, F. A., Siemiginowska, A., Smith, R. K., \& Wise, M. 2006, in Society of Photo-Optical Instrumentation Engineers (SPIE) Conference Series, Vol. 6270, Society of Photo-Optical Instrumentation Engineers (SPIE) Conference Series

Galloway, D. K., Cumming, A., Kuulkers, E., Bildsten, L., Chakrabarty, D., \& Rothschild, R. E. 2004, ApJ, 601, 466

Galloway, D. K., Lin, J., Chakrabarty, D., \& Hartman, J. M. 2010, ApJ, 711, L148

Galloway, D. K., Muno, M. P., Hartman, J. M., Psaltis, D., \& Chakrabarty, D. 2008, ApJS, 179, 360

Galloway, D. K., Psaltis, D., Chakrabarty, D., \& Muno, M. P. 2003, ApJ, 590, 999

Gottwald, M., Haberl, F., Parmar, A. N., \& White, N. E. 1986, ApJ, 308, 213

Greenhill, J. G., Giles, A. B., \& Coutures, C. 2006, MNRAS, 370 , 1303

Haberl, F., Stella, L., White, N. E., Gottwald, M., \& Priedhorsky, W. C. 1987, ApJ, 314, 266

Hanawa, T. \& Sugimoto, D. 1982, PASJ, 34, 1

Houck, J. C. \& Denicola, L. A. 2000, in Astronomical Society of the Pacific Conference Series, Vol. 216, Astronomical Data Analysis Software and Systems IX, ed. N. Manset, C. Veillet, \& D. Crabtree, 591

in 't Zand, J. J. M., Jonker, P. G., \& Markwardt, C. B. 2007, A\&A, 465, 953

in 't Zand, J. J. M. \& Weinberg, N. N. 2010, A\&A, submitted (arXiv.org:1001.0900)
King, I. R., Stanford, S. A., Albrecht, R., Barbieri, C., Blades, J. C., Boksenberg, A., Crane, P., Disney, M. J., Deharveng, J. M., Jakobsen, P., Kamperman, T. M., Macchetto, F., Mackay, C. D., Paresce, F., Weigelt, G., Baxter, D., Greenfield, P., Jedrzejewski, R., Nota, A., Sparks, W. B., \& Sosin, C. 1993, ApJ, 413, L117

Kuster, M., Kendziorra, E., Benlloch, S., Becker, W., Lammers, U., Vacanti, G., \& Serpell, E. 2002, ArXiv Astrophysics e-prints

Lattimer, J. M. \& Prakash, M. 2007, Phys. Rep., 442, 109

Lewin, W. H. G., van Paradijs, J., \& Taam, R. E. 1993, Space Sci. Rev., 62, 223

Madej, J., Joss, P. C., \& Różańska, A. 2004, ApJ, 602, 904

Martí, J., Mirabel, I. F., Rodriguez, L. F., \& Chaty, S. 1998, A\&A, 332, L45

Misanovic, Z., Galloway, D. K., \& Cooper, R. L. 2010, ApJ, 718, 947

Nakajima, T., Iwamuro, F., Maihara, T., Motohara, K., Terada, H., Goto, M., Iwai, J., Tanabe, H., Taguchi, T., Hata, R., Yanagisawa, K., Iye, M., Kashikawa, N., \& Tamura, M. 2000, AJ, 120, 2488

Nelemans, G. 2008

Nobili, L., Turolla, R., \& Lapidus, I. 1994, ApJ, 433, 276

Piro, A. L. \& Bildsten, L. 2007, ApJ, accepted (astro-ph/0704.1278)

Priedhorsky, W. \& Terrell, J. 1984, ApJ, 284, L17

Schatz, H., Aprahamian, A., Barnard, V., Bildsten, L., Cumming, A., Ouellette, M., Rauscher, T., Thielemann, F.-K., \& Wiescher, M. 2001, Physical Review Letters, 86, 3471

Stella, L., White, N. E., \& Priedhorsky, W. 1987, ApJ, 312, L17

Strohmayer, T. E., Zhang, W., Swank, J. H., Smale, A., Titarchuk, L., Day, C., \& Lee, U. 1996, ApJ, 469, L9

Weinberg, N. N., Bildsten, L., \& Schatz, H. 2006, ApJ, 639, 1018 